\title{
Thermal poling of fibers with multi-anodes
}

\author{
Huang, Lin; An, Honglin; Hayashi, Juliano G.; Ren, Guobin; Stefani, Alessio; Fleming, Simon
}

Published in:

Bragg Gratings, Photosensitivity and Poling in Glass Waveguides and Materials, BGPPM 2018

Link to article, DOI:

10.1364/BGPPM.2018.BTh2A.4

Publication date:

2018

Document Version

Peer reviewed version

Link back to DTU Orbit

Citation (APA):

Huang, L., An, H., Hayashi, J. G., Ren, G., Stefani, A., \& Fleming, S. (2018). Thermal poling of fibers with multianodes. In Bragg Gratings, Photosensitivity and Poling in Glass Waveguides and Materials, BGPPM 2018 [BTh2A.4] Optical Society of America (OSA). Optics InfoBase Conference Papers Vol. Part F98-BGPPM 2018 https://doi.org/10.1364/BGPPM.2018.BTh2A.4

\section{General rights}

Copyright and moral rights for the publications made accessible in the public portal are retained by the authors and/or other copyright owners and it is a condition of accessing publications that users recognise and abide by the legal requirements associated with these rights.

- Users may download and print one copy of any publication from the public portal for the purpose of private study or research.

- You may not further distribute the material or use it for any profit-making activity or commercial gain

- You may freely distribute the URL identifying the publication in the public portal 


\title{
Thermal Poling of Fibers with Multi-anodes
}

\author{
Lin Huang, ${ }^{1,2}$ Honglin An, ${ }^{1}$ Juliano G. Hayashi, ${ }^{1}$ Guobin Ren, ${ }^{2}$ Alessio Stefani, ${ }^{1,3}$ and Simon Fleming ${ }^{1 *}$ \\ ${ }^{1}$ Institute of Photonics and Optical Science (IPOS), School of Physics, The University of Sydney, NSW, 2006, Australia \\ ${ }^{2}$ Key Lab of All Optical Network \& Advanced Telecommunication Network of EMC, Beijing Jiaotong University, Beijing 100044, China \\ ${ }^{3}$ DTU Fotonik, Department of Photonics Engineering, Technical University of Denmark, DK-2800 Kgs. Lyngby, Denmark \\ Author e-mail address: simon.fleming @ sydney.edu.au
}

\begin{abstract}
We demonstrate thermal poling of fibers with $\sim 50$ and $\sim 500$ anodes. The second order nonlinearity layers are developed surrounding all the rings of wires in the $\sim 50$ anode fiber and the outer rings of the $\sim 500$ anode fiber.

OCIS codes: (190.4160) Multiharmonic generation; (190.4370) Nonlinear optics, fibers; (190.4400) Nonlinear optics, materials; (160.3918) Metamaterials.
\end{abstract}

\section{Introduction}

Thermal poling can break macroscopic centrosymmetry of silica glasses and create second-order nonlinearity (SON), $\chi^{(2)}$, in glasses and waveguides. The mechanism is generally considered to be the migration of mobile charges, which are driven by the electric field at elevated temperature. However, in the two-anode poled twin-hole fibers, when the two electrodes are at the same positive voltage, a counterintuitive observation was obtained that a much stronger and temperature stable SON layer was created between the two anodes [1,2]. While the electric field should be small between the two anodes when the same potential is applied, an avalanche-like positive feedback mechanism happens during the two-anode poling process [1]. As fibers can accommodate more holes and thus, more electrodes, it is interesting to explore how far this thermal poling can be extended from just two anodes, and the extent to which an induced SON can be distributed through the volume of the glass. Poling these structures and inducing a SON may enable tunable metamaterials [3] with potential applications in sub-diffraction imaging and waveguide devices. In this paper, we demonstrate experimentally thermal poling of fibers with $\sim 50$ and $\sim 500$ tin wire array anodes, arranged in concentric rings. SON layers are created surrounding the wires. Simulations of the thermal poling process based on a two-dimensional charge dynamics model $[4,5]$ are conducted to illuminate the physical mechanism involved.

\section{Fabrication and thermal poling of multi-wire array optical fiber}

The wire array metamaterial fibers based on glass and tin are fabricated by the stack and draw technique. The glass is AR-GLAS from Schott and its chemical composition in weight is: $\mathrm{SiO}_{2}(69 \%), \mathrm{B}_{2} \mathrm{O}_{3}(1 \%), \mathrm{K}_{2} \mathrm{O}(3 \%), \mathrm{Al}_{2} \mathrm{O}_{3}(4 \%)$, $\mathrm{Na}_{2} \mathrm{O}(13 \%), \mathrm{BaO}(2 \%), \mathrm{CaO}(5 \%), \mathrm{MgO}(3 \%)$. The diameter of the fiber and the tin filled holes are $\sim 1000 \mu \mathrm{m}$, and $\sim 10 \mu \mathrm{m}$, respectively, and the pitch of the tin filled holes is $\sim 20 \mu \mathrm{m}$. During poling the fibers are placed on an electrically grounded hotplate at high temperature. To avoid thermal runaway and dielectric breakdown, the poling voltage is increased from $500 \mathrm{~V}$ to $1.8 \mathrm{kV}$ with a step of $100 \mathrm{~V}$ every $2 \mathrm{~min} .1 .8 \mathrm{kV}$ DC voltage is applied to the tungsten wire for $30 \mathrm{~min}$. The $\sim 50$ and $\sim 500$ wire fibers were poled at $210^{\circ} \mathrm{C}$, and a second $\sim 50$ wire fiber was poled at $250^{\circ} \mathrm{C}$ for comparison. After poling, the samples are cooled down to room temperature before the voltage was switched off. The poled samples were characterized using second harmonic ( $\mathrm{SH}$ ) microscopy [6]. The resolution was estimated to be $\sim 0.4 \mu \mathrm{m}$.

Typical SH microscope images of the $\sim 50$ wire fiber poled at $1.8 \mathrm{kV}, 210^{\circ} \mathrm{C}$ for a duration of $30 \mathrm{~min}$ are shown in Fig. 1. Figure 1(a) shows that SH signals were found in rings around most of the holes. Several holes are not well poled, presumably due to poor connection of the wires. SH signals were clearly enhanced when poled at higher temperature, as shown in Fig. 1 (b). The intensities of the SH signals in Fig. 1 (b) are comparable to those observed in poled silica fiber. Note that since y-polarized light is used in the SH microscopy, the observed SH signals are strong along the $\mathrm{y}$-direction while weak along the $\mathrm{x}$-direction. For the poled $\sim 500$ wire fiber at $210^{\circ} \mathrm{C}, 1.8 \mathrm{kV}$ and $30 \mathrm{~min}$ duration (not shown here) the SH signals are induced in the glass proximal to the wires making up the outer rings of wires with intensity stronger than the results in Fig. 1(a) but lower than that in Fig. 1(b).

The results show that the SH signals are generated just underneath the surface of the holes, and after a poling period of 30 min they have a width of $\sim 1 \mu \mathrm{m}$. This observation, combined with the strong dependency of SH intensity on poling temperature, is consistent with the accepted mechanism of poling (rather than perhaps being due to polarization in the metal glass interface). More specifically we can conclude that the alkali metal ions are the dominant charges migrating during poling. The enhancement of the SH signal intensity at higher temperature is also consistent with short poling duration results, during which the depletion region is formed gradually, and the migration of the following second kind of mobile charges can be neglected. 
The results shown in Fig. 1 are apparently quite anomalous, because if all the wires are at the same potential it might be expected that the outer ring of wires would effectively screen the inner wires and there would be no field within this ring, and just radial fields from the outer ring. This observed pattern however can be explained by referring to the avalanche-like positive feedback mechanism [1] or the similar "self-adjustment" mechanism [2]. The migration of charges starts from the outer wire rings, where the strength of the electric field is initially relatively strong, this process creates local depletion regions in which the resistivity is very high. This in turn causes a modification of the electric field distribution around the inner rings, and promotes the migration of charges in the inner rings. Our simulation based on two dimensional charge dynamics model confirms the self-adjustment mechanism, and shows the SON layers are induced from the outer rings to the inner rings.
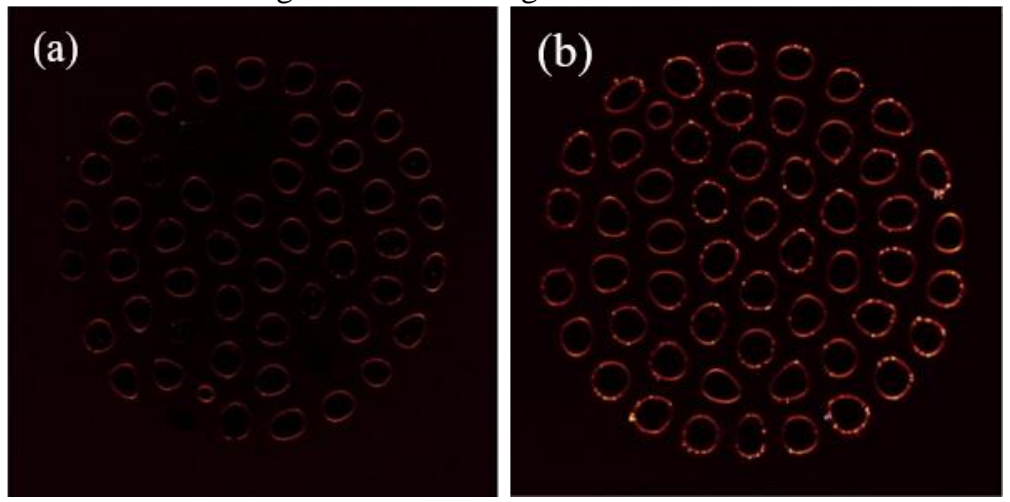

Fig. 1. SH signals of the $\sim 50$ wire array anode poled fiber (a) at $210^{\circ} \mathrm{C}, 1.8 \mathrm{kV}$ for $30 \mathrm{~min}$ and (b) at $250^{\circ} \mathrm{C}, 1.8 \mathrm{kV}$ for $30 \mathrm{~min}$. Note that the tin (melting temperature $\sim 232^{\circ} \mathrm{C}$, ) is liquid at poling temperature $250^{\circ} \mathrm{C}$, potentially improving the continuity of the tin wires in the fiber.

\section{Conclusion}

In conclusion, we demonstrate experimentally poling $\sim 50$ and $\sim 500$ tin wire array fiber. The results show the $\sim 50$ wire fibers are successfully poled such that all rings of wires develop SON layers, and the SON increases with increased poling temperature. SON layers were also observed surrounding the outer rings of wires in the $\sim 500$ wire fiber poled at lower poling temperature. Our simulation based on a two-dimensional charge dynamics model reveals the charge migration process in the multi-wire fiber. Although there is initially no electric potential drop in the inner wire rings, which would prevent charge migration in this region, the SON layers develop at the outer rings of wire at first, then the inner rings, and finally all wires are surrounded by an induced SON layer after sufficiently long poling time. This is consistent with the self-adjustment mechanism in a two-anode poled fiber.

The findings extend poling of fiber from two anodes to multi anodes. Poled multi-wire fiber can be potentially applied for electro-optic modulation or frequency conversion, even a QPM structure may be obtained by using the multi-wire glass material from the side if the wires are removed. In addition, these results may also open up possibilities for tunable metamaterials.

\section{Funding}

National Natural Science Foundation of China (NSFC) (61178008); ARC Discovery Projects (DP130102348 \& DP140104116); Marie Sklodowska-Curie grant of the European Union's Horizon 2020 research and innovation programme (708860).

\section{References}

[1] W. Margulis, O. Tarasenko, and N. Myrén, "Who needs a cathode? Creating a second-order nonlinearity by charging glass fiber with two anodes," Opt. Express 17(18), 15534-15540 (2009).

[2] H. An and S. Fleming, "Investigating the effectiveness of thermally poling optical fibers with various internal electrode configurations," Opt. Express 20(7), 7436-7444 (2012).

[3] S. Fleming, A. Stefani, X. Tang, A. Argyros, D. Kemsley, J. Cordi, and R. Lwin, "Tunable metamaterials fabricated by fiber drawing," J. Opt. Soc. Am. B 34(7), D81-D85 (2017).

[4] A. Camara, O. Tarasenko, and W. Margulis, "Study of thermally poled fibers with a two-dimensional model," Opt. Express 22(15), 1770017715 (2014).

[5] L. Huang, G. Ren, and X. Sun, "Evolution of Two-dimensional Electric Field in Poled Twin-hole Optical Fiber," in International Photonics and OptoElectronics Meetings, OSA Technical Digest (online) (Optical Society of America, 2014), FTh2F.5.

[6] H. An, S. Fleming, and G. Cox, "Visualization of second-order nonlinear layer in thermally poled fused silica glass," Appl. Phys. Lett. 85(24), 5819-5821 (2004). 